\title{
Nitrogen Enables the Intensity Modulation of Charge Transfer and Spin Paramagnetism in Graphdiyne
}

Mingjia Zhang, ${ }^{\dagger},{ }^{\#}$ Zhaoyong Guan, ${ }^{\dagger,}$ Z Ze Yang, ${ }^{\dagger}$ Xiuli Hu, ${ }^{\dagger}$ Xiaoxiong Wang, ${ }^{\S}$ Yunze Long, ${ }^{*} \S$ and Changshui Huang ${ }^{*},+, / 1$

${ }^{\dagger}$ Qingdao Institute of Bioenergy and Bioprocess Technology, Chinese Academy of Sciences, Qingdao 266101, P.R. China

${ }^{*}$ School of Chemistry and Chemical Engineering, Shandong University, Jinan, 250100, P. R. China

${ }^{\S}$ College of Physics, Qingdao University, Qingdao 266071, P.R. China

"Center of Materials Science and Optoelectronics Engineering, University of Chinese Academy of Sciences, Beijing 100049, P.R. China 


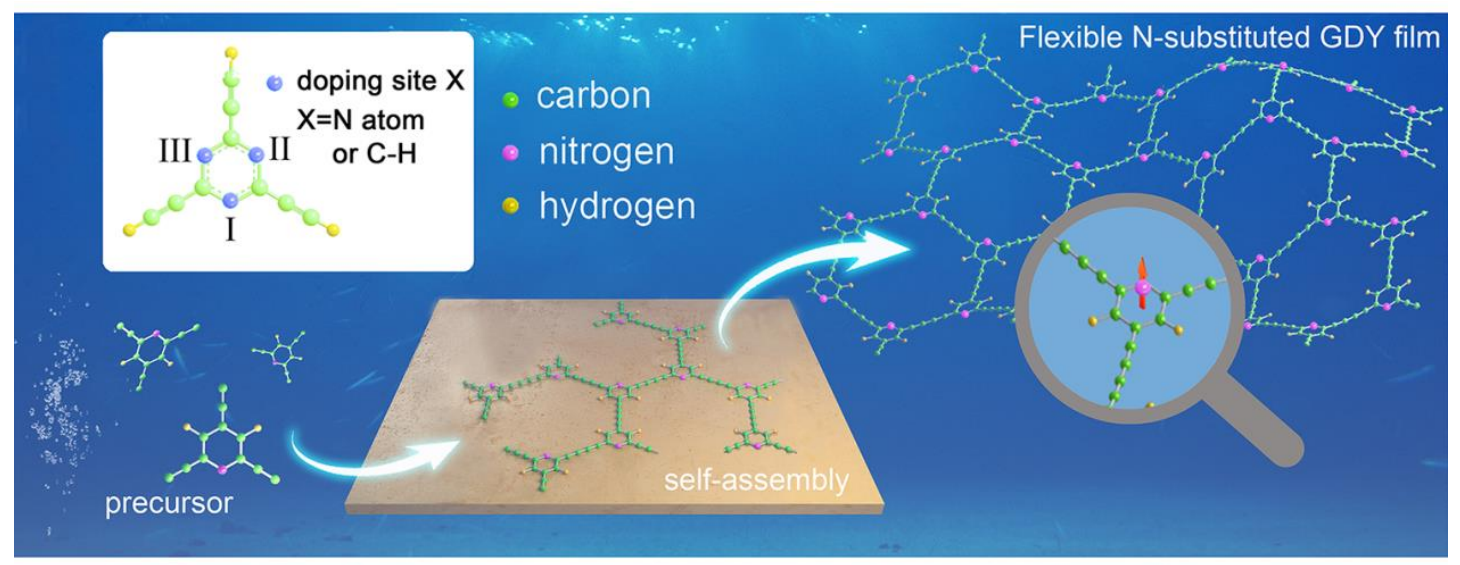

Figure S1. Synthetic route diagram of atomic-scale nitrogen-doped GDY based on monomer design strategy.

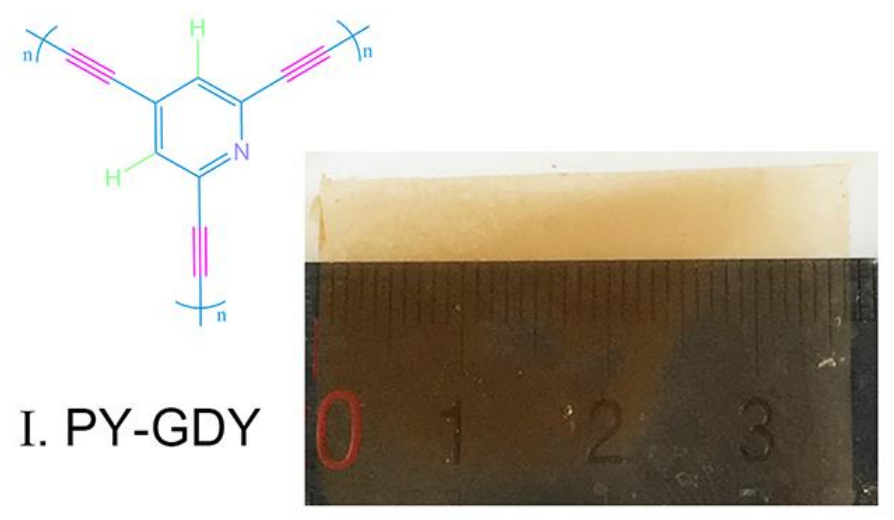

Figure S2. Structural representation of PY-GDY and the corresponding optical images of synthesized films.

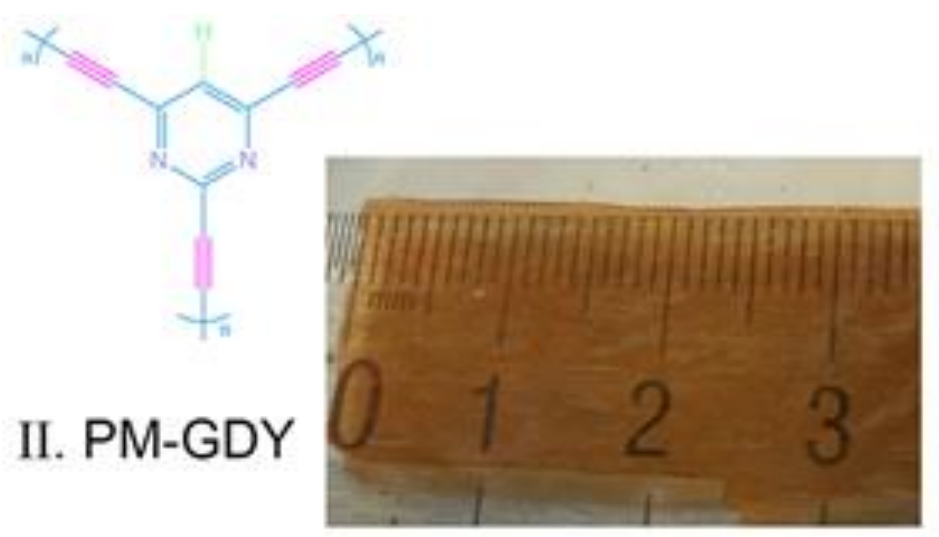

Figure S3. Structural representation of PM-GDY and the corresponding optical images of synthesized films. 


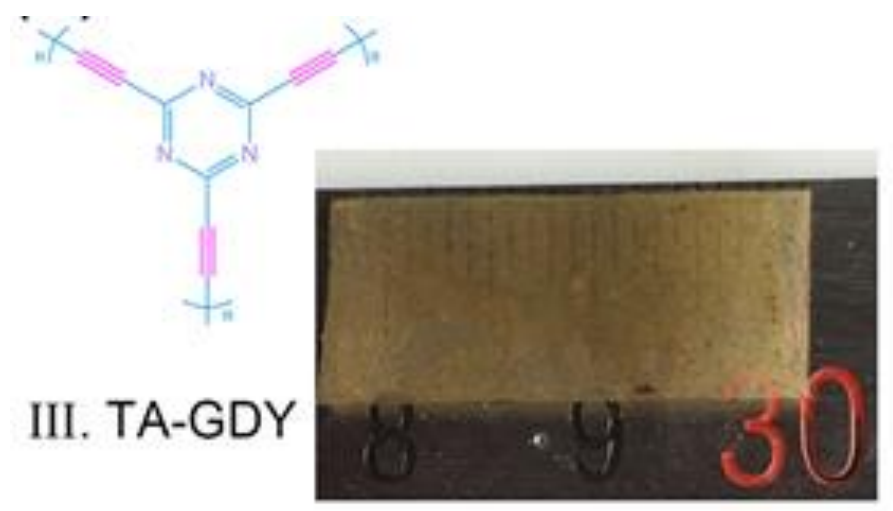

Figure S4. Structural representation of TA-GDY and the corresponding optical images of synthesized films.

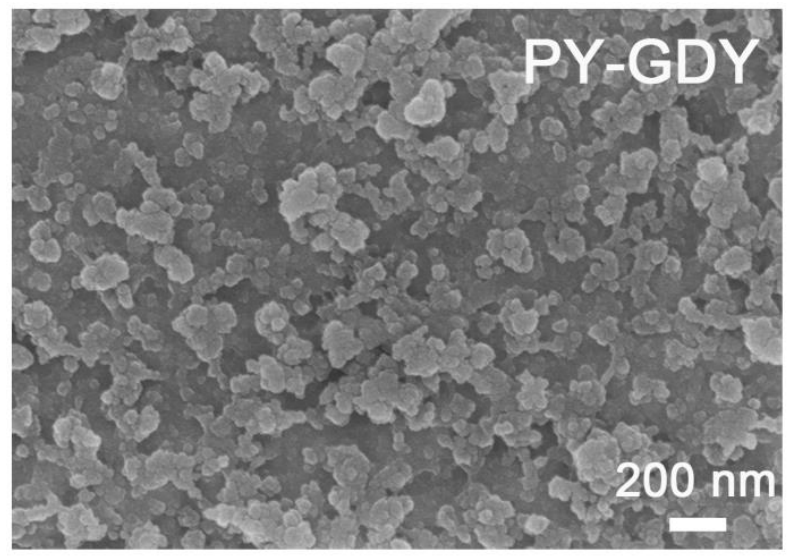

Figure S5. SEM image of PY-GDY.

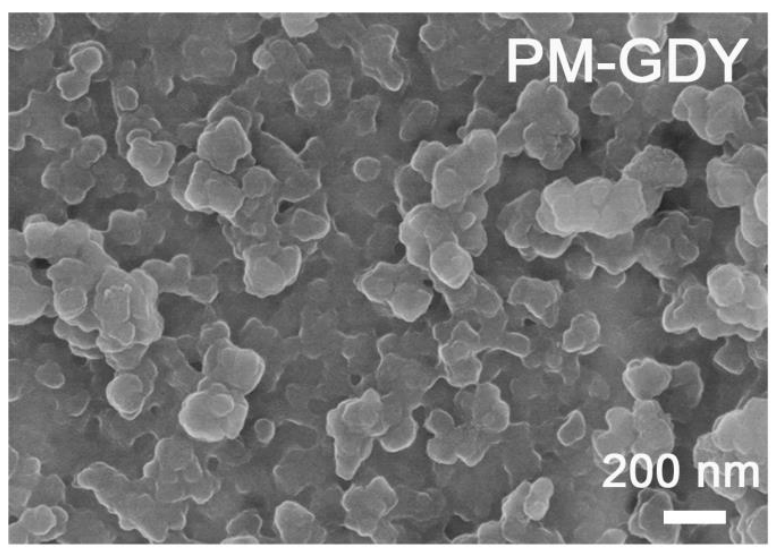

Figure S6. SEM image of PM-GDY. 


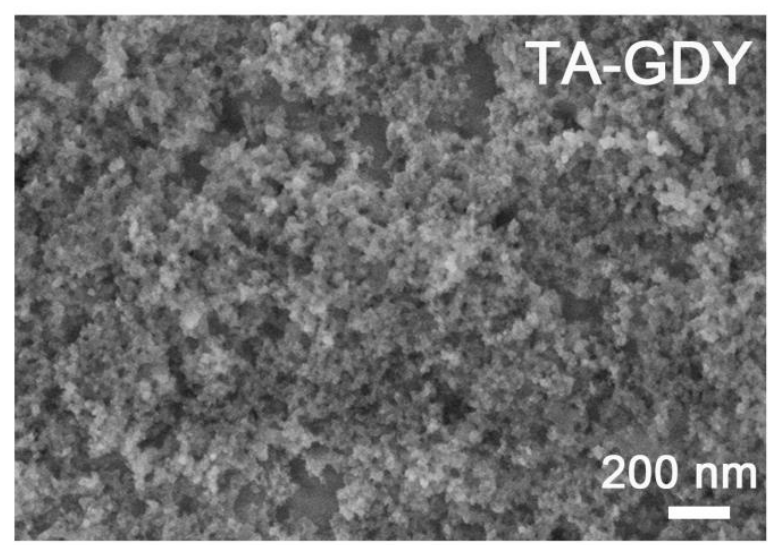

Figure S7. SEM image of TA-GDY.

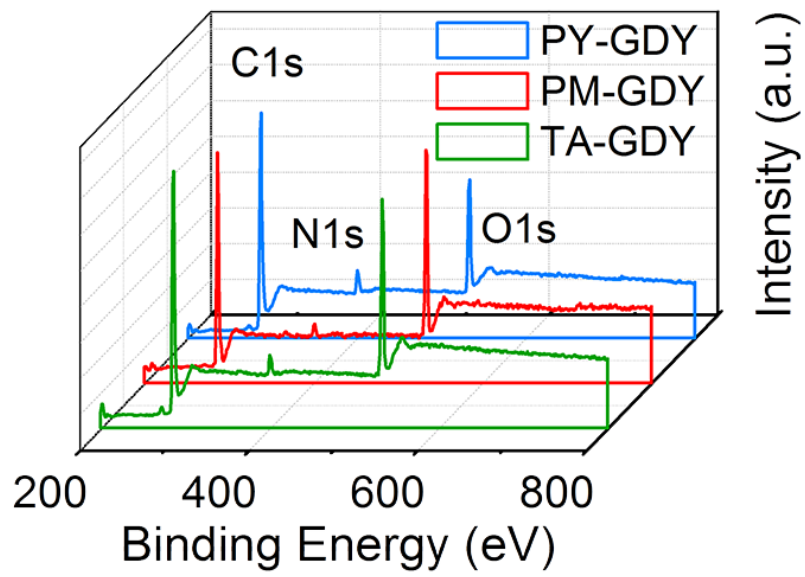

Figure S8. XPS spectra of PY-GDY, PM-GDY, and TA-GDY. 


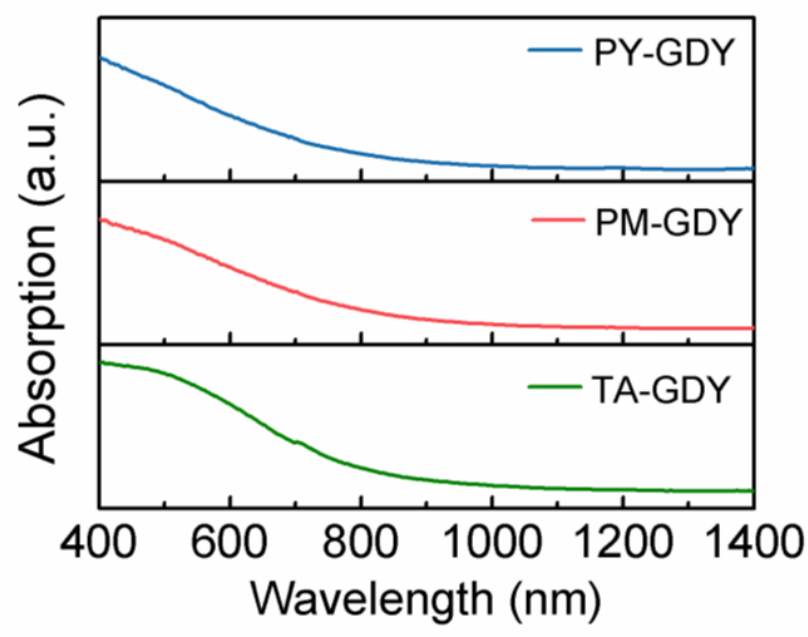

Figure S9. UV-vis spectra of N-doped GDY.

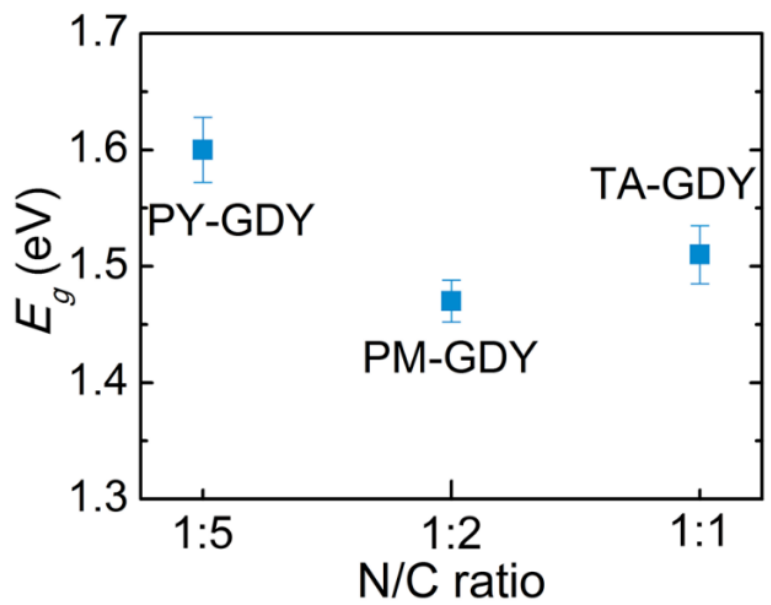

Figure S10. Band gap of nitrogen-doped graphdiyne with different nitrogen-carbon ratios.

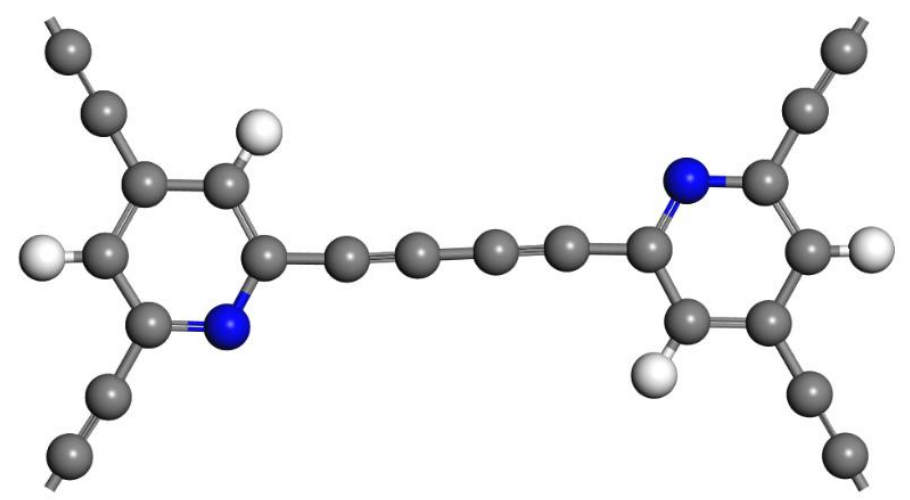

Figure S11. Top view of AA stacked PY-GDY two-layer model. 

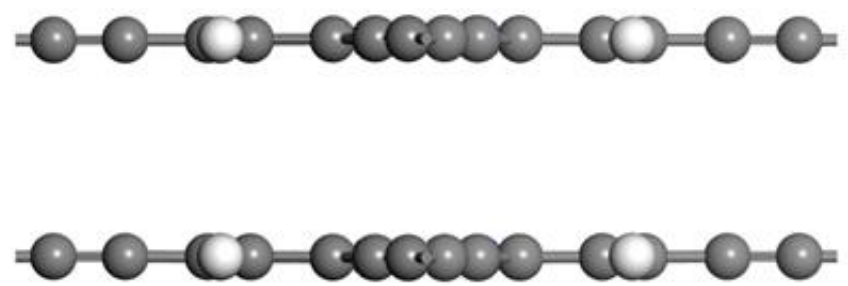

Figure S12. Side view of AA stacked PY-GDY two-layer model.

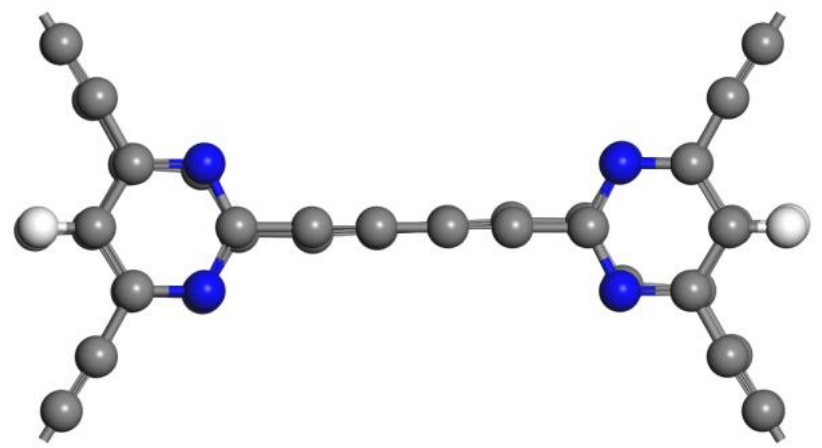

Figure S13. Top view of AA stacked PM-GDY two-layer model.
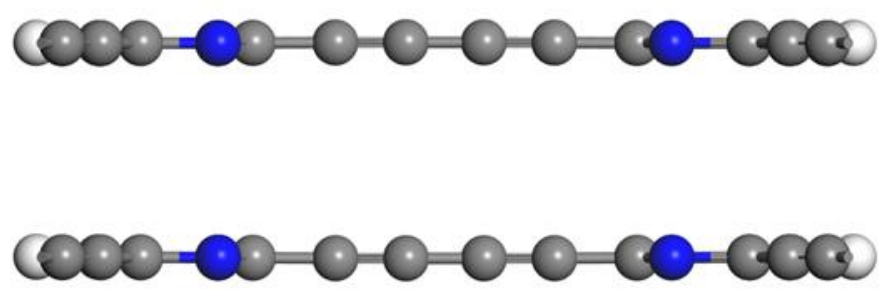

Figure S14. Side view of AA stacked PM-GDY two-layer model.

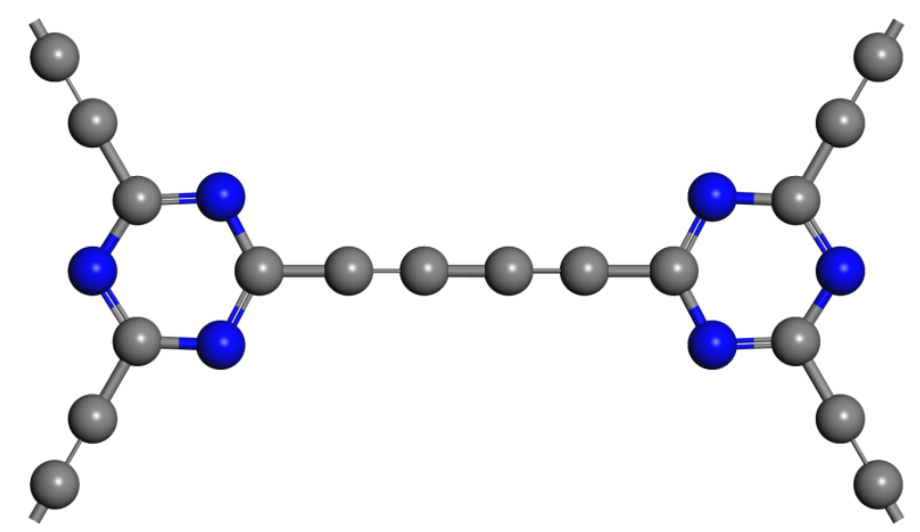

Figure S15. Top view of AA stacked TA-GDY two-layer model. 

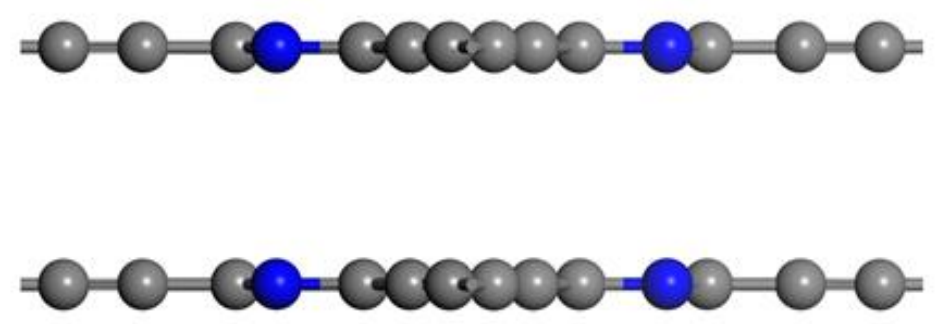

Figure S16. Side view of AA stacked TA-GDY two-layer model. 

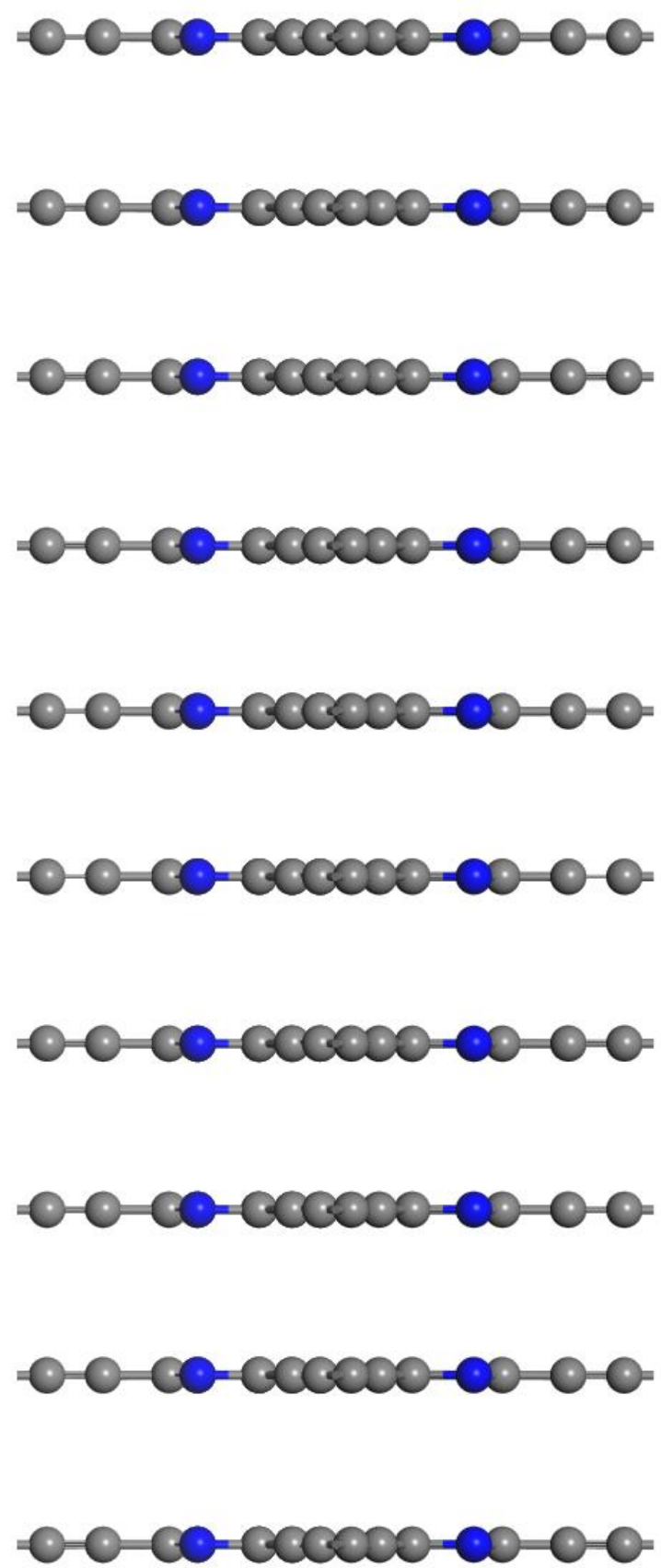

Figure S17. Side views of AA stacked TA-GDY 10-layer model. 


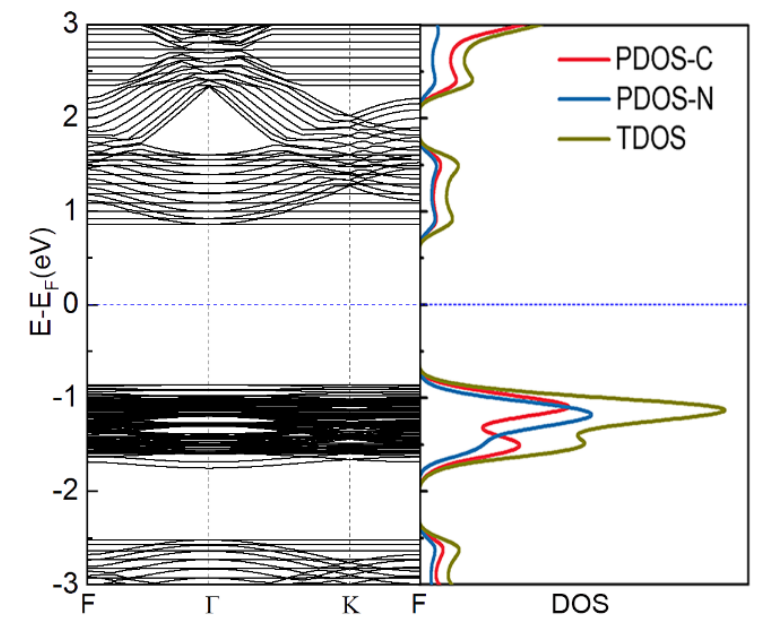

Figure S18. Band structures and DOS of ten-layer TA-GDY with AA stacking using the PBE function.

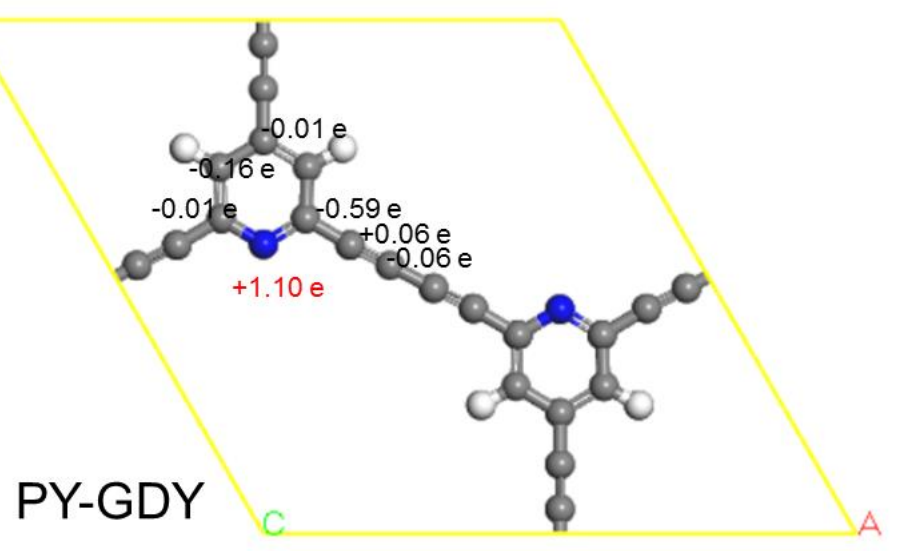

Figure S19. Charge transfer by Bader analysis for PY-GDY.

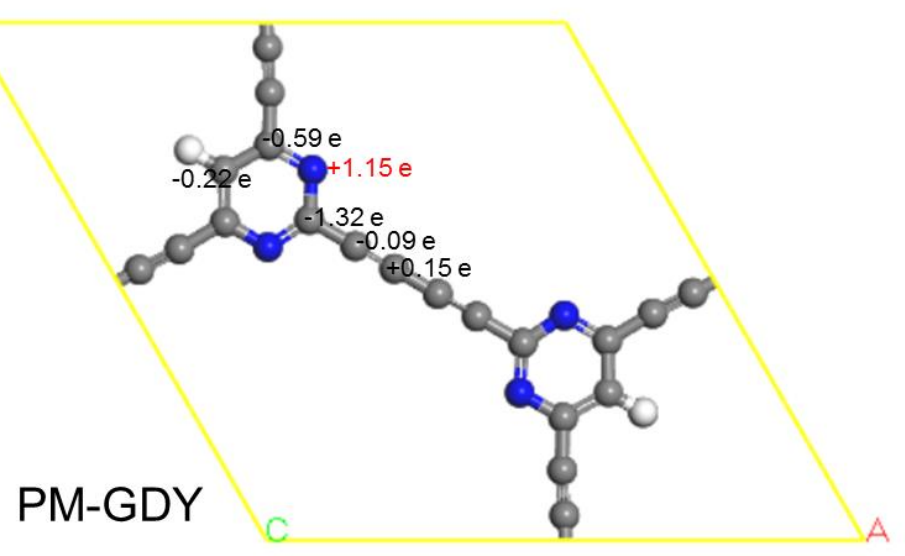

Figure S20. Charge transfer by Bader analysis for PM-GDY. 


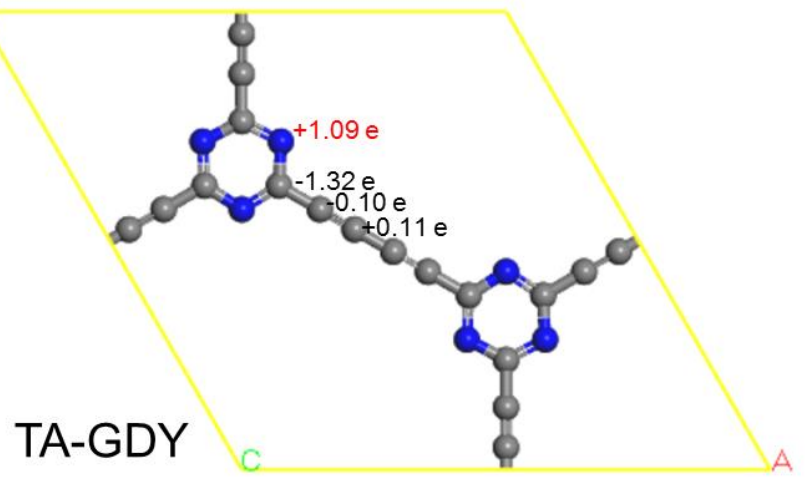

Figure S21. Charge transfer by Bader analysis for TA-GDY. 